\title{
Las familias homoparentales en las políticas sociales de salud
}

\author{
Homoparental families in health policies and legal regulations: the Cuban \\ case
}

Yailyn Rosales Sanchez. ${ }^{1}$ \& Luis Efraín Velasteguí López. ${ }^{2}$

DOI: https://doi.org/10.33262/visionariodigital.v5i2.1691

\begin{abstract}
:
Introduction: Since January 1959, the social policies in Cuba, have had a consistent approach with the socialist principles of the Revolution. One of the visible social groups is the family, even when there is no social policy focused directly on their demands as an institution or place for intervention. The existing programs have to do with the needs of certain members of the community, such as children, pregnant women, the elderly and people with disabilities. They also protect and institutionalize a nuclear and heterosexual family model, but models which break with this vision are left out. It is the case of homoparental families. Objectives: The research aims at describing the management given to homoparental families by the social health policies and legal regulations in Cuba. Methodology: It is mainly a qualitative study in which the content analysis was used to obtain the information. Results: The concepts of family and marriage in Cuba protect the union between a man and a woman. Social policies respond to the interests of nuclear and heterosexual families. Homoparental families continue to be on the margins of social programs and policies, which brings about inequalities to these families.
\end{abstract}

Key words: social policies; social health policies; homoparental families.

\section{Resumen:}

Introducción: Las políticas sociales en Cuba, desde enero de 1959, han tenido un enfoque coherente con los principios socialistas de la Revolución. Uno de los grupos sociales visibilizados es la familia, incluso cuando no existe una política social enfocada

\footnotetext{
${ }^{1}$ Licenciada en Sociología. Especialista del Departamento de Investigación y Docencia del Centro Nacional de Educación Sexual (CENESEX) Email: yrosaless@infomed.sld.cu, http// orcid.org/00000002-7120-9237

${ }^{2}$ Ciencia Digital Editorial, Ecuador, luisefrainvelastegui@cienciadigital.org
} 
directamente a sus demandas como institución o espacio de intervención. Los programas existentes responden a las necesidades de determinados miembros de la misma, como es el caso de los niños, las mujeres embarazadas, los ancianos y personas con discapacidad. Además amparan e institucionalizan un modelo de familia nuclear y heterosexual, donde quedan fuera aquellos modelos que rompen con esta visión. En esta situación se encuentran las familias homoparentales. Objetivos: La investigación tiene como objetivo describir el tratamiento que reciben las familias homoparentales desde las políticas sociales y las normativas legales en Cuba. Metodología: El estudio es esencialmente cualitativo y para la obtención de información se aplicó el análisis de documentos. Resultados: Los conceptos de familia y matrimonio en Cuba amparan la unión entre un hombre y una mujer. Las políticas sociales responden a los intereses de las familias nucleares y heterosexuales. Las familias homoparentales continúan al margen de los programas y políticas sociales, lo cual trae consigo que se generen desigualdades.

Palabras claves: políticas sociales; políticas de salud; familias homoparentales.

\section{Introducción:}

Las políticas sociales en Cuba, desde enero de 1959, han tenido un enfoque universal y coherente con los principios socialistas de la Revolución. Se han direccionado a suplir necesidades básicas identificadas, como es el caso de la salud, la educación, la vivienda y la seguridad social. Todo este proceso ha estado en consonancia con los cambios en los que ha estado inmersa la sociedad cubana. (Marquetti, 2001) Uno de los grupos sociales en los que han impactado es la familia, aunque en nuestro país no existe una política social enfocada directamente a ella, que responda a sus necesidades como institución. Las proyecciones han estado direccionadas hacia determinados miembros de la misma, como es el caso de los niños, las embarazadas, los ancianos y las personas con discapacidad. Existen un grupo de programas que se implementan en este sentido, tales como "Educa a tu hijo", "Maternidad y Paternidad responsables", "Ley de la maternidad de las trabajadoras" y los programas dedicados a tratar los problemas de fertilización de la pareja. (Colectivo de autores, 2010; Álvarez, 2014)

En los procesos de implementación de las mismas se puede constatar que están orientados a un modelo de familia nuclear y heterosexual, donde quedan fuera aquellos modelos que rompen con esta visión. Una de las tipologías de familia que no cuenta con esa visibilización en el ámbito legal e institucional es la homoparental. Estas tienen sus propias dinámicas internas, costumbres, sistemas de valores, modelos de crianza, contradicciones y necesidades básicas, que pueden coincidir o no con las de otras familias. Desde el ámbito legal igualmente han estado invisibilizadas, ya que no se reconocen como un modelo de familia o tipo de unión, solo es legalmente aceptada entre un hombre y una mujer. La revisión de estas políticas y normativas legales debe atemperarse a la sociedad actual, donde confluyen varios modelos de familia, en pos de erradicar la desigualdad que se genera producto de esta invisibilización. Hacerlas 
visibles permite concientizar sobre la necesidad de crear una política que tenga a la familia como actor principal y que contemple todas las tipologías que se vivencian en nuestra sociedad. Por tanto, la investigación tiene como objetivo describir el tratamiento que reciben las familias homoparentales desde las políticas sociales y las normativas legales en Cuba.

\section{Metodología:}

Para desarrollar la revisión bibliográfica se aplicó el analisis de documentos, como técnica de investigación de la naturaleza del discurso. Con el empleo de la misma se pretende realizar una decripción precisa y lógica del contenido comunicativo de la documentación revisada, lo cual permite comprender el proceso que se analiza. En este sentido se realizó una revisón de las investigaciones que han tratado el tema de las políticas sociales de salud y cómo se han acercado a las familias homoparentales. Además, se analizaron los artíulos de la Constitución de la República de Cuba que hacen referencia a los conceptos de familia y matrimonio. Para realizar esta revisión se establecieron como categorías de análisis el concepto de familia que se valida en los documentos legales y políticas sociales y el tratamiento a las familias homoparentales.

\section{Resultados:}

El modelo de desarrollo cubano ha tenido como pilares básicos la promoción del desarrollo social y humano, la equidad y la justicia social, sobre la base de la eliminación de las vulnerabilidades. Sus resultados en términos de desarrollo social, reducción de la pobreza y promoción de la mujer son indiscutibles. No obstante, algunas tendencias emergieron o se profundizaron en el contexto de la crisis y la reforma económicas -diferenciación socioeconómica y desigualdad social, expresándose en la polarización de los ingresos y la aparición de grupos vulnerables. La necesidad de profundizar y ampliar los estudios sobre los temas de pobreza respecto a aquellos sectores más vulnerables, a fin de favorecer formas diversas de promoción y protección social, argumentan la relevancia científica del estudio. Cuba presenta además una posición favorable según el Índice de Pobreza Humana, con logros significativos en la situación social de determinados miembros de la familia y una elevada protección social mediante los instrumentos jurídicos y la seguridad social. Esto se conjuga con una alta prevalencia de jefatura femenina de hogar, el creciente envejecimiento poblacional, la visibilización de brechas sociales debido a la aparición de nuevos actores sociales con diversidad de ingresos, la aparición de nuevas tipologías de familias, así como por algunos indicadores de vulnerabilidad en las mujeres, problemáticas que también resultan relevantes desde el punto de vista social y humano. (Zabala, 2009)

Uno de los grupos sociales que han vivenciado el alcance de estas políticas sociales es la familia, aun cuando no haya una política de familia en sí. Este impacto se ha materializado en programas de salud, educación sexual y familiar, orientación a la mujer y la familia, priorizando a miembros específicos (ancianos, niños, niñas y adolescentes, mujeres, personas con discapacidad). Estos programas responden a un modelo de 
familia nuclear y heterosexual, por tanto, en el proceso de implementación quedan fuera todas aquellas que rompen con esta idealización. Uno de estos modelos son las familias homoparentales, conformadas por una pareja del mismo sexo y su (s) descendencia (s), ya sea de uno de sus miembros o sean guardadores de hecho, con la cual pueden tener o no lazos de consanguinidad o convivencia con esta descendencia en un espacio común. Entre sus miembros existen relaciones afectivas que regulan su comportamiento y su relación con el contexto. (5) En tal sentido, el Grupo de Estudios sobre la Familia del Centro de Investigaciones Psicológicas y Sociológicas (CIPS) en su libro "Las familias cubanas en el parteaguas de dos siglos" (2010) recomienda a las instancias pertinentes legalizar y visibilizar la situación de las familias constituidas por parejas del mismo sexo, y establecer medidas que garanticen sus derechos; además de eliminar toda práctica o normativa jurídica discriminatoria derivada de la orientación sexual de las personas. (2)

Las familias homoparentales desde las normativas legales en Cuba:

El Ordenamiento Jurídico vigente en Cuba adolece de una producción legislativa suficiente con respecto al reconocimiento de las familias homoparentales y es evidente la carencia de acciones legales eficaces que les permitan acceder a la formación de una familia por la vía legal. Además, no ofrece una cobertura que garantice el reconocimiento y ejercicio pleno de los derechos sexuales de un modo inclusivo. En este sentido es importante señalar que existen instituciones que trabajan a favor del reconocimiento y ejercicio pleno de todos los derechos de los ciudadanos. Una de estas instituciones es el Centro Nacional de Educación Sexual (CENESEX), el cual tiene como misión asesorar al Ministerio de Salud Pública en cuanto a la elaboración de políticas sociales que aborden la educación integral de la sexualidad y el reconocimiento y garantía de los derechos sexuales, sin ninguna manifestación de discriminación. (6)

En función de estos procesos transformadores, se llevó a cabo el análisis de la Constitución de la República por parte de la Asamblea del Poder Popular en sesión extraordinaria los días 21 y 22 de julio de 2018, donde se plantearon importantes reformas en el sentido de actualizarla en función de que responda a los intereses de la sociedad de nuestro tiempo. Se aprobó el 24 de febrero de 2019, antecedido por un proceso de consenso popular, donde cada persona pudo plantear sus criterios en cuanto a los artículos que recoge. Uno de los cambios más importantes hace referencia al proceso de formación de la familia como un derecho humano y que recibe el reconocimiento del Estado cubano. Otro de los cambios está relacionado con el artículo que regula el concepto de matrimonio, el cual es entendido como unión entre dos personas, dándole un cambio a la intencionalidad del mismo, reconociéndolo como una de las formas de organización de las familias; además de la inclusión en su Artículo 42 de la protección ante cualquier forma de discriminación por orientación sexual o identidad de género. Estas modificaciones devienen en un proceso de consulta popular, que demuestra el carácter democrático y participativo del Estado cubano. Con estas trasformaciones a la Carta Magna se pone de manifiesto el interés político por lograr la inclusión de las personas sin discriminación por orientación sexual o identidad de 
género, pero es importante continuar trabajando en aras de que la sociedad se sensibilice en este sentido, ya que, el contar con los basamentos legales es solo un paso en el largo camino de la erradicación de la violencia y discriminación hacia aquellas personas que no siguen los modelos tradicionales de ser hombre o mujer.

Por su parte, El Código de Familia vigente ratifica en sus conceptos el binarismo hombre-mujer, lo cual contraviene la mayor ley del país, La Constitución. La noción de matrimonio y unión matrimonial muestra su carácter heterosexual al esclarecer que dichas uniones se reconocerán solamente entre hombre y mujer. Derivadas de esta concepción se establecen una serie de terminologías aludiendo a las relaciones conyugales, recogidas en el documento:

Unión matrimonial: se refiere al hecho por el cual, un hombre y una mujer, con aptitud legal, (contraen) consienten voluntariamente en unirse para hacer vida en común, de manera estable y singular, independientemente de que legalicen el hecho de acuerdo con lo establecido en la ley. (7)

Matrimonio formalizado: se refiere al acto mediante el cual un hombre y una mujer, con aptitud legal, concurren voluntariamente y de manera consciente ante un funcionario facultado para ello y dejan legalizada su decisión de unirse en matrimonio o dejan legalizada la unión matrimonial contraída por ellos en fecha anterior retrotrayendo sus efectos. (7)

Matrimonio reconocido: se refiere al acto mediante el cual, una persona (hombre o mujer) concurre ante el tribunal competente para que éste, mediante resolución judicial, reconozca que entre esa persona que ante el tribunal insta y otra (fallecida o viva) existió una unión matrimonial que contrajeron voluntariamente y con aptitud legal en fecha anterior. (7)

El concepto de matrimonio esclarece que solo es legal la unión entre un hombre y una mujer, limitando la legalización de aquellas uniones que rompen con estos patrones. Resulta una interpretación heterosexual del concepto, lo cual permite constatar la verticalidad que caracteriza la construcción de la ley. Se puede percibir que esta es el resultado de la concepción moral válida para la clase que detenta el poder. Está fuertemente arraiga en esta conceptualización la antiquísima mirada discriminatoria a la homosexualidad, aun cuando ya podemos hablar de países que aprueban las uniones homosexuales y de una sociedad cubana más preparada en cuanto a los temas de diversidad sexual.

Como resultado de las necesidades actuales de la familia cubana y la confluencia de diversos modelos, este código está siendo sometiendo a un proceso de revisión y posterior modificación de algunos de sus artículos. La Constitución establece el plazo de dos años a partir de su aprobación para realizar el proceso de consulta y referendo del proyecto de Código de Familia, lo cual queda recogido en las disposiones transtorias de la Constitución de la República. El reconocimiento de las uniones consensuales entre personas del mismo sexo constituye una de las modificaciones que se están sometiendo 
a análisis. Todo este proceso de revisión queda explicado en la Gaceta oficial 006 extraordinaria de 15/1/2008, emitida por la Asamblea Nacional del Poder Popular, declarando en su Primer por cuanto que las disposiciones recogidas en el Código de Familia vigente han requerido y requieren un proceso de reformulaciones para atemperarse a los cambios de la sociedad cubana actual. (8)

Las familias homoparentales: un reto para las políticas sociales de salud

La realidad contemplada en el aspecto legal en cuanto al reconocimiento de una tipología de familia abarca los modelos de políticas sociales, las cuales están bastante alejadas de la realidad cotidiana de las personas que deben ser beneficiadas con dichas políticas. Jelin (2005) resalta que la organización familiar debe ser tomada como eje de análisis, fundamentalmente a la hora de elaborar estas prácticas. Los modelos actuales giran en torno a las funciones del mercado, las responsabilidades del estado, de qué se encarga la familia y las condiciones de las actividades comunitarias. En muchas ocasiones la responsabilidad de la familia consiste en suplir las fallas del estado y este, a su vez, se enfoca en revertir, en lo posible, las diferencias que impone el mercado y hacerse responsable de algún miembro de la familia porque esta carece de los recursos. (9)

Arriagada (2001) plantea que una política que favorezca a la familia como eje central debe combinar varias características, como son la subsidiariedad y la participación, donde el Estado, en un primer momento garantice mediante subsidios las capacidades de las mismas, lo cual derive en mayor participación de estas en el ámbito público y solucionar sus problemas. Otro aspecto a tener en cuenta es la integralidad, la que asegura un tratamiento abarcador a las necesidades de todos los miembros de la familia. Pero en la práctica no sucede así, las políticas se enfocan en la subvención a miembros específicos, constituyen acciones para erradicar la pobreza y con un enfoque microsocial, a corto plazo, asistencialista y fragmentario. El objetivo fundamental de estas políticas debe estar encaminado a fortalecer a la familia como sujeto de derecho y poner mayor atención en las familias más vulnerables. (10) Las familias homoparentales constituyen en la actualidad uno de estos grupos que no se visibilizan en estas políticas, ya que rompen con los modelos avalados socialmente, donde la unión hombre-mujer establece el arquetipo ideal. La contemplación de sus problemáticas en las políticas sociales ha suscitado interesantes polémicas y debates en el ámbito donde se conforman las mismas. En la década de los 90 comienzan a verse los primeros adelantos para la legalización de la situación de estas familias en muchos países.

En el caso de Cuba el análisis debe partir de la no existencia de una política social que tome a la familia como agente de intervención; todas tienen un enfoque universal, con la mirada orientada hacia miembros de la misma, como son los niños, las mujeres embarazadas, los ancianos y las personas con discapacidades. La Dra. Mayda Álvarez señala que el enfoque de las políticas hacia las familias tiene cinco objetivos diferentes: la regulación del matrimonio, la convivencia y la creación de condiciones para la 
formación de parejas; las orientadas a garantizar y asegurar la fecundidad; las que regulan la relación familia-trabajo. (3)

Las familias homoparentales quedan al margen de estas proyecciones, al romper con el modelo tradicional. El hecho de no estar representados en los programas de atención a la familia y, por ende, no ser considerados como tal, coloca a estos modelos en situaciones de vulnerabilidad en diversos sectores de la sociedad, como es el caso de la salud. Estaban encaminados a la concreción de determinadas estructuras que reforzaban la existencia de un modelo ideal de familia. Respondían al orden económico y los valores culturales de la clase dominante, el cual avalaba el tipo de familia nuclear y heterosexual, aun cuando no se planteaba de manera explícita. La realidad actual supera estas proyecciones, ya que confluyen en la sociedad varios modelos de familia que rompen con este paradigma y hace que las políticas sociales se replanteen su alcance. Una de estas familias es la homoparental, que pone en jaque mate estas acciones para legitimar un modelo ideal de familia.

El sistema de salud en Cuba, aun cuando se caracteriza por la accesibilidad y calidad en los servicios, todavía se encuentra mediado por los preceptos del sistema patriarcal, delimitando la postura asignada para hombres y mujeres, emergiendo la subjetividad de los que ofrecen el servicio cuando estos modelos se distorsionan. Diversas investigaciones han colocado la mirada en este asunto, tomando como muestra de las mismas a familias homoparentales, formadas por dos mujeres o dos hombres. Además, han incorporado al análisis la perspectiva del personal de salud que implementa estos programas y su preparación a la hora de tratar a las mismas. En aquellas que han tomado como muestra un grupo de familias homoparentales integradas por mujeres lesbianas, la mayoría ha percibido la existencia de un distanciamiento en el momento de recibir atención especializada, específicamente en el examen físico por parte del personal de salud, influenciado por la presencia de prejuicios portados por los mismos, por lo que limitan su asistencia al médico por temor a declarar su historial sexual. En las ocasiones que han acudido en busca de ayuda profesional, se han percatado que existe desinformación y prejuicios encubiertos por parte del personal de salud respecto al conocimiento del funcionamiento de las relaciones eróticas afectivas entre mujeres lesbianas. (11)

Actualmente la formación profesional con conocimiento de la vida sexual de las personas con orientación sexoerótica homosexual constituye un elemento invisible en las políticas de salud, al igual que el tema de la orientación sexual como comportamiento social a tener en cuenta en los procesos salubristas, dado por la inadecuada formación de recursos humanos y de cobertura de información acerca de la salud sexual de las mujeres lesbianas en los servicios médicos, estructurado sobre un modelo androcéntrico y homofóbico. (11)

Colocar la mirada en este sentido permite hacer señalamientos a las políticas y programas sociales que incluyen a la familia, y hacer más equitativas las acciones de salud, en aras de concretar un servicio público que desmonte todos los patrones 
heterosexistas que emergen al respecto, incluso contribuye a erradicar las brechas de vulnerabilidad que pueden resultar de estos enfoques.

Dentro de los programas orientados a la familia está el relacionado con la atención de las parejas infértiles. El Programa para la Atención a la Pareja Infértil establece un protocolo de atención que trata de estandarizar prácticas, diagnósticos y tratamientos, pero que excluye la exploración de la vivencia y el padecer de la pareja, lo que además se ve reforzado por los puntos de encuentro según la perspectiva de género de prestadores y usuarios de los servicios. (12)

Algunos resultados de las investigaciones que han tomado este programa como objeto de análisis refieren que la reproducción social y los espacios donde se desarrolla el individuo, son los que determinan en última instancia las percepciones individuales y colectivas de la función reproductiva y la infertilidad. Aunque el espacio social de las mujeres se ha ampliado más allá del hogar y la maternidad ha ocupado otro lugar, todavía la sociedad sigue esperando de ella que sea madre. El hombre en muchas ocasiones, sufre la infertilidad desde la demanda social más que desde el propio deseo de ser padre. La implicación en el proceso se ve muchas veces comprometida, siendo la mujer la que asiste con mayor frecuencia a los servicios de salud. La presión social continúa siendo un factor determinante en el afrontamiento de estos procesos. En el país se destaca en este sentido

En función de este aspecto, algunas investigaciones han destacado la importancia de entender la subjetividad de los usuarios de este servicio de salud. Uno de los estudios más recientes, desarrollado por la Universidad de Ciencias Médicas de Santiago de Cuba, se centró en analizar el componente psicológico que influye en las parejas que asisten a estas consultas. La muestra quedó constituida por una pareja, legalmente constituida, la cual llevaba alrededor de 2 años intentando concebir un hijo, por lo cual era tratada desde hacía un año en la consulta de infertilidad. La esposa era educadora de círculo infantil y el esposo ejercía la actividad del cuentapropismo. Uno de los hallazgos de la investigación confirma una de las ideas citadas con anterioridad, donde la incidencia de la mirada social condiciona la presencia de ambos miembros de la pareja en las consultas, lo que ha incidido en la realización del tratamiento y en la permanencia en la consulta de infertilidad. (13)

Se debe hacer un análisis psicológico del impacto de la infertilidad para las personas que se encuentran en esta situación y la repercusión para la vida en pareja. Además, estos estudios destacan la implicación de la familia en este proceso, como garante de apoyo y sustento ante esta situación. Es importante resaltar que, aunque estos estudios han comenzado a ver el proceso más allá de un padecimiento, retomando su implicación social para las personas que acuden al servicio, este protocolo está diseñado o priorizan a las parejas heterosexuales, quedando fuera las mujeres solteras y las familias que rompen con la fórmula hombre-mujer. En su mayoría, aplican los estudios de casos para analizar varias aristas del proceso, destacando solo parejas heterosexuales, además de hacer alusión en todo momento a este tipo de unión. 
Otro de los centros que ha impulsado investigaciones sobre el tema de la atención a las parejas infértiles es el Centro Territorial de Atención a la Pareja Infértil de la provincia de Cienfuegos. Uno de los estudios realizados contó con una muestra de 92 parejas (hombre-mujer) que solicitaron el servicio en este centro. Dentro de los resultados del mismo se destaca la idea de que la infertilidad está estrechamente vinculada al sexo femenino, viendo el proceso de concepción como una responsabilidad exclusiva de las condiciones biológicas de las mujeres. En los modelos que se aplican en el sistema de salud para tratar estos prejuicios, se delimitan los roles que juegan los miembros de la pareja: rol de marido o mujer dañado por sentirse inferior o que no cumple con su cometido social. (14)

Aun cuando estas investigaciones hayan logrado dirigir la mirada hacia el componente social de este padecimiento, más allá de un problema de salud, se observa la presencia de parejas heterosexuales en estas consultas. Es importante señalar que se cuenta con la voluntad política para priorizar la atención a la salud, con la consolidación de un modelo gratuito, con calidad y al alcance de todas las personas en los diferentes niveles del sistema. Pero son destacables a su interior brechas de desigualdad, ya que se prioriza la atención a la infertilidad de las parejas heterosexuales, quedando fuera aquellas que no cumplen con este requisito o personas que de manera independiente desean acceder al servicio de salud.

Se debe tener en cuenta la incidencia de la subjetividad del personal de salud a la hora de atender a las personas que no se asumen como heterosexuales, que rompen con el binomio hombre-mujer o que analizan a la familia desde otra perspectiva, más allá del modelo tradicional. Tomando como bandera la igualdad que garantizan las leyes sobre la salud en Cuba y los cambios que se están experimentando en la familia cubana actual, se hace necesaria una revisión de todas las normativas jurídicas que rigen a la sociedad. Dentro de ellas se encuentran las del Sistema de Salud y sus programas, el cual debe atemperarse a estas transformaciones, desde un análisis de las potencialidades y limitaciones del programa para incluir a las familias homoparentales, como una de estas nuevas tipologías.

\section{Conclusiones:}

- Las políticas sociales dirigidas a la familia están pensadas en un modelo nuclear y heterosexual, obviando las tipologías que confluyen en la sociedad contemporánea. En Cuba no existe una política de familia, sino acciones dirigidas a grupos sociales específicos, igualmente orientadas al modelo avalado socialmente. Quedan fuera aquellas tipologías que rompen con este ideal de familia.

- El hecho de que las familias homoparentales no estén contempladas en las políticas sociales y carezcan de apoyo legal, las coloca en una situación de desventaja, lo cual influye en su accesibilidad a los servicios.

- En Cuba se cuenta con la voluntad política para priorizar la atención a la salud, con la consolidación de un modelo gratuito, con calidad y al alcance de todas las 
personas en los diferentes niveles del sistema. Pero son destacables a su interior brechas de desigualdad, ya que se prioriza la atención a la infertilidad de las parejas heterosexuales, quedando fuera aquellas que no cumplen con este requisito o personas que de manera independiente desean acceder al servicio de salud.

\section{Referencias bibliográficas}

Marquetti Nodarse, H. Cuba: Reformas estructurales en los años noventa. Resultados, problemas y perspectivas. La Habana: Centro de Estudios de la Economía Cubana; 2001.

Colectivo de autores. Las familias cubanas en el parteaguas de dos siglos. Colombia: D’vinni; 2010.

Álvarez, M. Familia y Género. Continuidad y rupturas. Colombia: Editorial Marada Trading; 2014.

Zabala, María del C. Jefatura femenina de hogar, pobreza urbana y exclusión social. Una perspectiva desde la subjetividad en el contexto cubano. Colección CLACSO; 2009.

Novales, José M. Parejas de hoy, familias del mañana... Estudio de la resiliencia en parejas homosexuales masculinas en La Habana. En Revista Sexología y Sociedad. Versión Electrónica. Edición 21; 2015.

Calaña, I.; Cubela, M. Memorias del 8vo Congreso de Educación, Orientación y Terapia Sexual. La Habana: CENESEX; 2018.

Mesa, Olga. Derecho de Familia. Facultad de Derecho, Universidad de La Habana, La Habana: 1997.

Asamblea Nacional del Poder Popular. Gaceta Oficial No. 006 Extraordinaria de 15 de enero de 2008. Cuba; 2008.

Jelin, E. Las familias latinoamericanas en el marco de las transformaciones globales: Hacia una nueva agenda de políticas públicas. Buenos Aires: CEPAL; 2005.

Arriagada, I. Familias latinoamericanas. Diagnóstico y políticas públicas en los inicios del nuevo siglo. Chile: CEPAL: 2001.

Frómeta, O. Salud sexual y desarrollo de la sexualidad de mujeres lesbianas, en edad adulta. En Revista Sexología y Sociedad. Número 19(2): 102 -115. Versión electrónica; 2013.

Díaz, Zoe; García Jordá, Daily. Cultura sobre la maternidad y la paternidad y su repercusión en la concepción de la infertilidad. (Referencia incompleta); 2010.

Rodríguez, María del C. Modelo vincular en la pareja tratada a causa de infertilidad. La Habana, 2016. 
Barco, Vladimir; Quintero, Carmen; Reyes, Aimé; Álvarez, Zoraida C. El modelo de adaptación ante la infertilidad de la pareja. (Referencia incompleta); 2014.

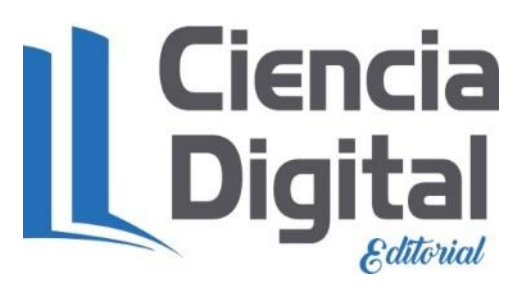




\section{PARA CITAR EL ARTÍCULO INDEXADO.}

Rosales Sanchez, Y., \& Velasteguí López, L. E. (2021). Las familias homoparentales en las políticas sociales de salud. Visionario Digital, 5(2), 137-148. https://doi.org/10.33262/visionariodigital.v5i2.1691

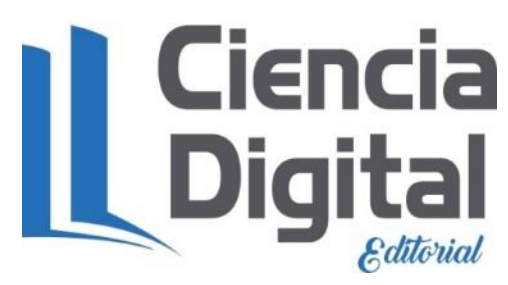

El artículo que se publica es de exclusiva responsabilidad de los autores y no necesariamente reflejan el pensamiento de la Revista Visionario Digital.

El artículo queda en propiedad de la revista y, por tanto, su publicación parcial y/o total en otro medio tiene que ser autorizado por el director de la Revista Visionario Digital.
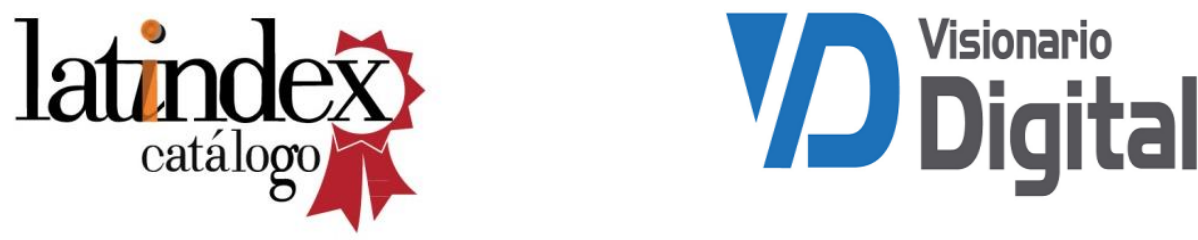